\title{
A novel spectrometer for spin-polarized electron energy-loss spectroscopy
}

\author{
H. Ibach ${ }^{\text {a) }}$ and D. Bruchmann \\ Institut für Schichten und Grenzflächen ISG3, Forschungszentrum Jülich, 52425 Jülich, Germany \\ R. Vollmer, M. Etzkorn, P. S. Anil Kumar, and J. Kirschner \\ Max-Planck Institut für Mikrostrukturphysik, Weinberg 2, 06120 Halle, Germany
}

(Received 5 March 2003; accepted 3 June 2003)

\begin{abstract}
By making use of advanced methods for the calculation of electron trajectories in the presence of space charge fields we have designed and built a novel electron energy-loss spectrometer for the purpose of measuring spin flip excitations at surfaces. The measurement of the spin asymmetry requires a deflection angle between the photocathode and the sample position which amounts to $90^{\circ}$ in total. That deflection angle is not provided by standard electron deflectors, or by a combination of those. We show that a $90^{\circ}$ deflection angle can be realized by a combination of two novel deflectors with $90^{\circ}$ and $180^{\circ}$ deflection angles each. The performance of the new spectrometer matches the performance of the best electron spectrometers developed for surface vibration spectroscopy. () 2003 American Institute of Physics. [DOI: 10.1063/1.1597954]
\end{abstract}

\section{INTRODUCTION}

Electron energy-loss spectroscopy (EELS) has established itself as one of the major tools for studying elementary excitations at solid surfaces. In particular for probing surface vibrations of adsorbed species and the dispersion of surface phonons the capabilities of EELS are unsurpassed by any other technique. The commercial availability of EELS instrumentation as developed in Jülich added greatly to its popularity. Present day spectrometers feature energy resolutions of less than $1 \mathrm{meV}$ in combination with a sensitivity which amounts to a small fraction of a monolayer for adsorbate vibrations. ${ }^{1-3}$ Progress in instrumentation became possible through the development of efficient numerical methods to calculate electron optical properties of energy dispersive devices beyond the limitations of the classical cylindrical and spherical deflectors, and of lens systems which are not radial symmetric. Novel energy dispersing elements compensate "spherical" aberrations to first order. ${ }^{3}$ Furthermore, the space charge aberrations on electron trajectories and the resulting limitations on the magnitude of the monochromatic current produced by energy dispersive elements are understood and can be taken into account to some degree. ${ }^{1}$ With these improvements the performance of EELS spectrometers is ultimately determined by the brightness of the available electron sources, which was the goal for the design of spectrometers formulated some 30 years ago. ${ }^{4}$

While advanced spectrometers leave little to be desired when probing the vibrational degrees of freedom they are lacking an important feature when it comes to electronic excitations. That is, the dependence of electronic excitations on the spin of the electron and electronic excitations which flip the spin could not be analyzed with satisfactory energy resolution. A particular desideratum is a technique for probing

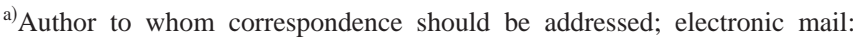
h.ibach@fz-juelich.de
}

surface and thin film magnons in the momentum space beyond the range accessible by optical techniques. ${ }^{5,6}$ The classical technique for measuring magnon dispersion, neutron scattering, by and large fails for surface and thin-film systems as it is lacking surface sensitivity. On the other hand, theoretical calculations show that the cross section for electron exchange scattering from magnons should be high enough to lead to detectable features in EELS, provided that one has means to discriminate them against other, non-spinflip, in particular vibrational excitations. ${ }^{7-9}$

In order to probe spin-flip events in EELS one makes use of negative electron affinity GaAs-photocathodes which emit partially polarized electron beams when illuminated by circular polarized light. ${ }^{10,11}$ The orientation of the spin is perpendicular to the cathode surface, i.e., longitudinal to the initial electron trajectory of the electrons when they are emitted from the cathode. For the purpose of measuring the spin asymmetry of surface excitations, the spin should be oriented perpendicular to the scattering plane and therefore transverse to the electron trajectory at the sample position. This requires a deflection of the electron trajectory of $90^{\circ}$ between the point of electron emission from the GaAs cathode and the sample. Unfortunately, a $90^{\circ}$ deflection is incompatible with the normal deflecting energy dispersive elements such as the cylindrical or spherical deflector which feature $127^{\circ}$ and $180^{\circ}$ deflection, in the absence of fringe field corrections. ${ }^{12}$ Initial approaches to the problem ${ }^{13}$ therefore introduced an additional deflector between the photocathode and electron monochromator. However, with the present understanding of the adverse effect of the space charge on the magnitude of the monochromatic current it is clear that such an additional deflection is likely to reduce the monochromatic current dramatically: High throughput spectrometers make effective use of the energy dispersion within the monochromator to reduce the aberrations caused by space charge. It would also be unwise to introduce the required additional deflecting element after the monochromator since such a deflection causes 
image aberration which would likewise have an adverse effect on the overall performance. The latter is because modern electron spectrometers require a good quality stigmatic imaging from the cathode all the way through the spectrometer to the detector. An optimum design for a spectrometer should therefore combine a $90^{\circ}$ deflection in the energy dispersive elements with stigmatic imaging of the entrance slit of the first monochromator onto the exit slit of the last analyzer of the entire spectrometer. While the classical concepts of energy dispersive electron deflectors fail to fulfill these requirements, the design described in Ref. 2 provides sufficient flexibility to accomplish the task. The solution to be described in this article features a two-stage monochromator, the first stage being a $90^{\circ}$ deflector which is followed by a $180^{\circ}$ deflector. The combination of both deflectors provides a (nearly) stigmatic image of the entrance slit onto the exit slit in the presence of space charge. The overall performance of the novel spectrometer matches the performance of the best spectrometer designed previously. ${ }^{14}$ For reasons to be discussed later it supercedes the performance of a spectrometer with a single $90^{\circ}$ deflector ${ }^{15}$ by far.

The article is organized as follows. The next section describes the basic concept of the new spectrometer as well as some of the technical details of the calculations in as much as they deviate from Ref. 1. In Sec. III the focal properties of the $90^{\circ}$ and the $180^{\circ}$ deflector with and without the effect of space charge are discussed. It is shown that the focal properties of the sequential arrangement of a $90^{\circ}$ and a $180^{\circ}$ deflector in combination with the aberrations induced by the space charge lead to a pseudo-stigmatic image at the exit slit as well as to an excellent resolution of the device. The overall performance of the spectrometer is discussed and compared with previous designs in Sec. IV.

\section{BASIC CONCEPT AND TECHNICAL ASPECTS}

The energy dispersing electrostatic deflectors used in the spectrometer are variants of the electrostatic deflector with aberration correction as introduced in $1993 .{ }^{14}$ These deflectors have a general cylindrical form of the deflector plates which are convexly curved around the central electron path (Fig. 1). ${ }^{16}$ The top and bottom of the deflector are covered by plates and the deflector has a metallic entrance and exit slit, so that the fringe field becomes an integral part of optical properties of the system. The deflector can be operated with the potential of the exit slit being reduced compared to the nominal pass energy. The system then works as a retarding deflector. By applying a negative "compression" bias to the top and bottom cover plates the focal length within the dispersion plane ( $x, y$ plane) is extended and shortened in the sagittal plane $(x, z$ plane) spanned by the electron trajectory and the $z$ axis perpendicular to the dispersion plane (Fig. 1). Stigmatic focusing can be achieved for a particular bias voltage and at a particular deflection angle. The total deflection angle at which stigmatic focusing is obtained depends on the ratio of the radii of the inner and outer deflection plates $\left(146^{\circ}\right.$ for the design introduced in 1993). The angular aberration coefficients of the deflector are controlled by the concave curvature of the deflection plates [Fig. 1(a)]. The radius of
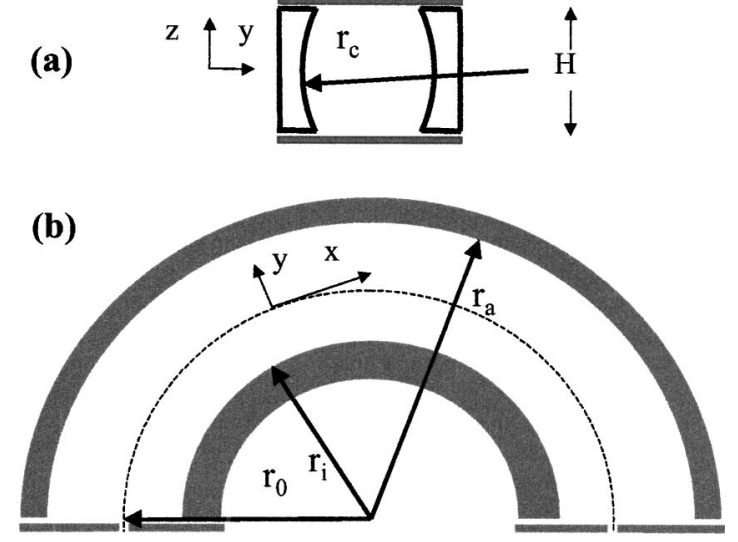

FIG. 1. Cross sections through the electrostatic deflector as used here. (a) displays the cross section which intersects the central path of the electrons at a right angle. The outer and inner deflecting plates have a concave curvature of a radius $r_{c}$. (b) shows the cross section in the energy dispersing plane for the $180^{\circ}$ deflector.

curvature $r_{c}$ is chosen such as to optimize the overall performance of the device when operating under space charge conditions.

The extra degree of freedom provided by the top and bottom cover plates can be used to make a deflector focusing within the dispersion plane for a wide range of deflection angles which encompass a $90^{\circ}$ as well as a $180^{\circ}$ deflection angle. The basic idea of the spectrometer described here is to use a double pass deflector consisting of a $90^{\circ}$ and a $180^{\circ}$ section, oppositely curved so that the total deflection angle becomes $90^{\circ}$. Biases on the top and bottom cover plates would be chosen such that within the dispersion plane trajectories emerging from a point in the entrance slit of the $90^{\circ}$ deflector are focused on the exit slit of the $180^{\circ}$ deflector, with an intermediate focus at the exit slit of the $90^{\circ}$ deflector. This is achieved by a positive and a negative bias $U_{B}$ on the top and bottom deflection plates of the $90^{\circ}$ and the $180^{\circ}$ deflector, respectively. The positive bias on the $90^{\circ}$ deflector leads to a diverging beam in the sagittal plane, the negative bias in the $180^{\circ}$ deflector to a focusing within the sagittal plane. The question to be explored is whether the construction can be made such that also within the sagittal plane trajectories emerging from a point in the entrance slit of the $90^{\circ}$ deflector are focused in the exit slit of the $180^{\circ}$ deflector (without an intermediate focus at the exit slit of the $90^{\circ}$ deflector). In that case the device would have the desired stigmatic focusing properties. This is what is being explored in detail with the help of numerical calculations of electron trajectories.

The overall design of the new spectrometer is displayed in Fig. 2. In addition to the combination of the $90^{\circ} / 180^{\circ}$ monochromator the spectrometer has a $146^{\circ}$ analyzer, as used in conventional electron energy-loss spectrometers. The illumination of the photocathode is by a laser diode with the beam passing through orifices in the outer deflecting plate of the $90^{\circ}$ deflector and the shielding of the lens system. The lens system again is as described in Ref. 1. The basic design parameters of the monochromator are summarized in Table I.

Calculations of electron trajectories involve a two step process. Step I is the solution of the Laplace equation (or the 


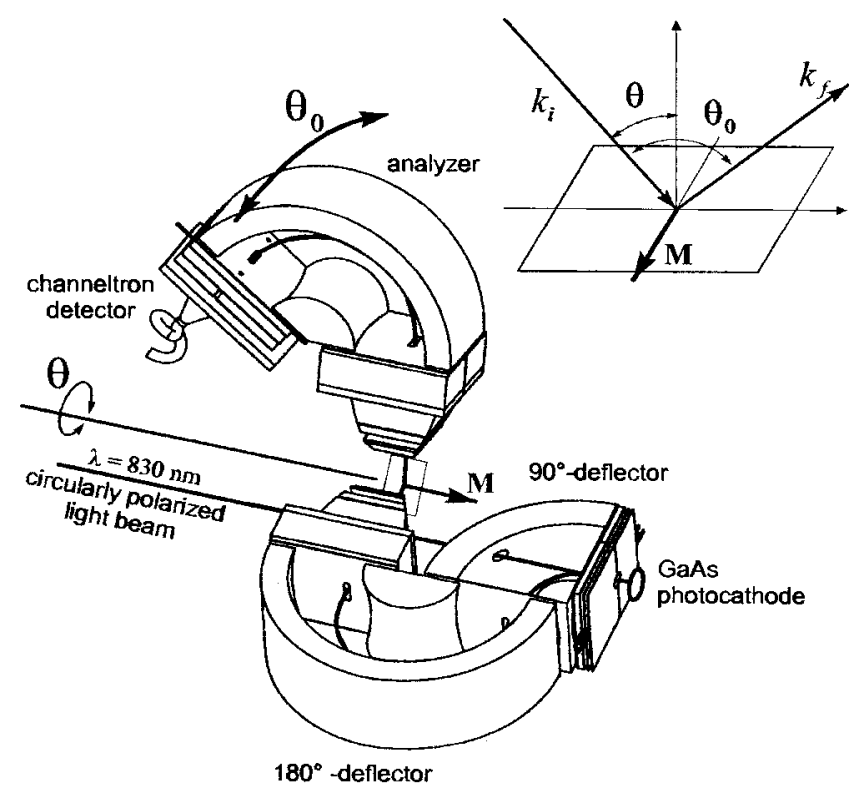

FIG. 2. Sketch of the electron spectrometer for spin dependent inelastic electron scattering. Electrons emitted from the photocathode are deflected in total by $90^{\circ}$ such that the spin is oriented perpendicular to the scattering plane at the sample (see inset). $k_{i}, k_{f}, \theta, \theta_{0}$, and $M$ denote the initial and final wave vector, the angle between $k_{i}$ and the surface normal, the angle between incoming and the emerging beam, and the magnetization of the sample, respectively.

Poisson equation if space charge is involved) and step II is the calculation of the electron paths. The computer time required for the second step exceeds the time required for the first step by a large factor, simply because one needs to calculate the trajectories of as many as $10^{5}$ electrons in order to determine resolution and transmission properties for each particular pass energy and each current load of the spectrometer. Efforts to develop or use efficient computer codes therefore pay off merely in the calculation of the electron trajectories. Because of the linearity of the Laplace equation, the Laplace equation needs to be solved only once for each spectrometer geometry and each independently variable potential. The actual potential distribution in the device is then calculated by a linear superposition. The Laplace equation was solved by the traditional though slow method of overrelaxation on a relatively coarse mesh. Electron trajectories are calculated by interpolating the potential between the grid points at each instantaneous position of the electron by a method which preserves the local differential properties of the Laplace equation. The Verlet algorithm is used to calculate the actual trajectories. For further details the reader is referred to Ref. 1.

Modern spectrometers operate under conditions at least of moderate space charge. For our particular problem the

TABLE I. Construction parameters of the $90^{\circ} / 180^{\circ}$ deflectors. See Fig. 1 for the notation.

\begin{tabular}{cccccc}
\hline \hline Deflector & $r_{c} / \mathrm{mm}$ & $r_{a} / \mathrm{mm}$ & $r_{i} / \mathrm{mm}$ & $r_{0} / \mathrm{mm}$ & $H / \mathrm{mm}$ \\
\hline $90^{\circ}$ & 25 & 60.3 & 20.3 & 42 & 48 \\
$180^{\circ}$ & 100 & 60.3 & 20.3 & 33.5 & 48 \\
\hline \hline
\end{tabular}
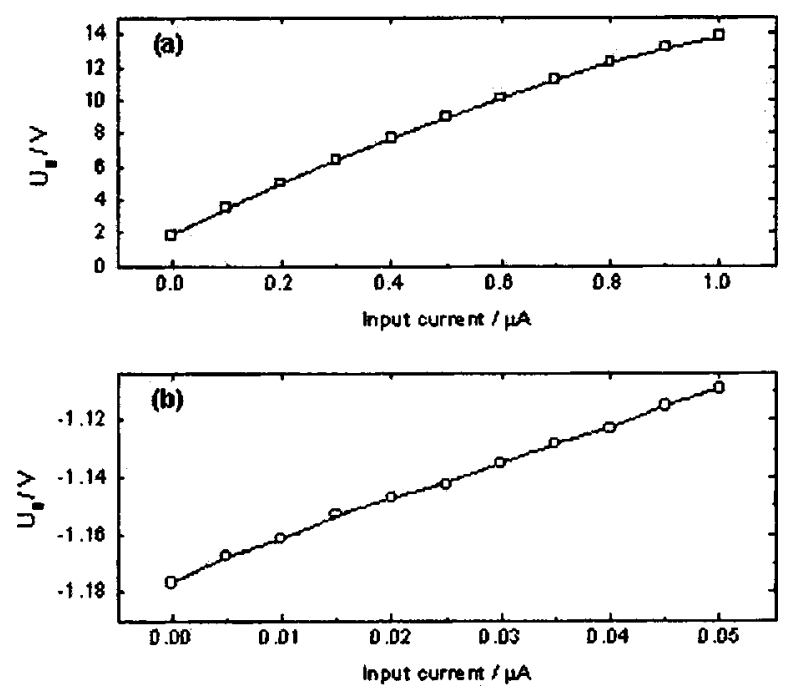

FIG. 3. Variation of the bias voltage $U_{B}$ necessary to keep the dispersion plane focus at the exit slit as a function of the input current. (a) For the $90^{\circ}$ deflector, (b) for the $180^{\circ}$ deflector. The reference level for $U_{B}$ is the voltage on the entrance and exit apertures. Data are calculated for deflection voltages $\Delta U=2 \mathrm{~V}$. For larger $\Delta U$ the $x$ axis scales up to larger currents. Note that $U_{B}$ is positive with respect to the pass energy for the $90^{\circ}$ deflector, and negative for the $180^{\circ}$ deflector.

space charge has an important effect on the formation of the stigmatic focus. The effect of space charge must therefore be taken into account with sufficient accuracy. To lowest order, the space charge can be determined by calculating the electron trajectories causing the space charge in the zero current limit. The potentials at the deflector are chosen such as to have the desired focal properties, e.g., a (stigmatic) focus at the exit slit. The space charge in the spectrometer is determined by feeding the spectrometer with a bundle of electrons which has the angular and energy distribution as provided by the cathode emission system or the previous deflector. The additional potential induced by the space charge is obtained by solving the Poisson equation in the deflector with all potentials on the electrodes set to zero. This potential is linear in the charge and thus linear in the current entering the deflector. It is therefore to be added to the potential arising from the solution of the Laplace equation for the deflecting plates. For a given, still small, current load the trajectories are then calculated again with the lowest order space charge potential, and the bias on the top and bottom cover plates is adjusted in order to keep the focal properties of the device as desired. The trajectories for the feeding bundle can then be calculated with the modified bias and a second iteration on the space charge is thereby obtained. For small currents, the first correction suffices, for higher currents more iterations may be needed. Technically, the appropriate number of iterations was achieved by raising the current in steps and calculating the space charge correction to the trajectories with the space charge potential obtained in the previous step. Figure 3 displays the bias voltage $U_{B}$ required to keep the focus in the dispersion plane at $90^{\circ}$ and $180^{\circ}$ deflection angle for increasing input currents. The bias $U_{B}$ is with reference to the voltage on the entrance and exit apertures. The deflection voltage is $\Delta U=2 \mathrm{~V}$ on both deflectors. The input currents are chosen to be in the range as used under typical operating conditions 

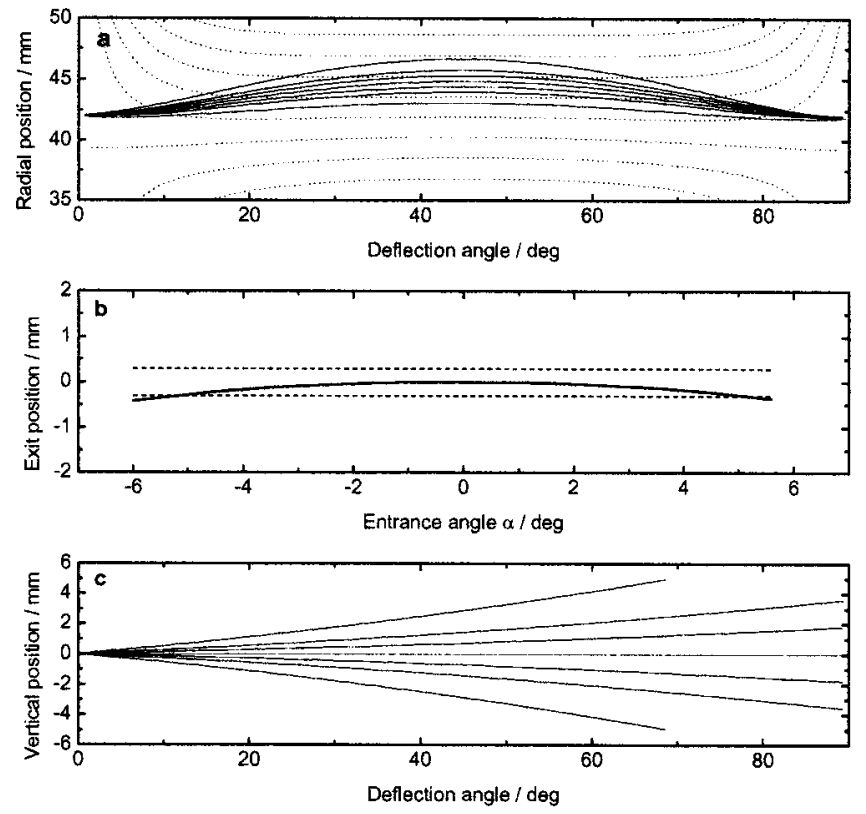

FIG. 4. Trajectories in the $90^{\circ}$ deflector in the zero current limit. (a) Solid and dashed lines denote trajectories and equipotential lines, respectively. (b) Radial position at the exit slit $y(\alpha)$ vs the entrance angle. Dashed lines denote the width of the exit slit. (c) Trajectories in the sagittal plane.

for $\Delta U=2 \mathrm{~V}$. For larger deflection voltages the current for a given focusing bias scales approximately as $(\Delta U)^{3 / 2}$.

\section{FOCUSING PROPERTIES OF THE $90^{\circ} / 180^{\circ}$ DEFLECTOR COMBINATION}

In this section we describe the focusing properties of the $90^{\circ}$ and $180^{\circ}$ deflector. The key results for the $90^{\circ}$ and $180^{\circ}$ deflector in the absence of space charge are presented in the three panels of Figs. 4 and 5, respectively. The biases $U_{B}$ on the top and bottom deflecting plates were set to have electrons emerging from a point in the entrance slit to focus
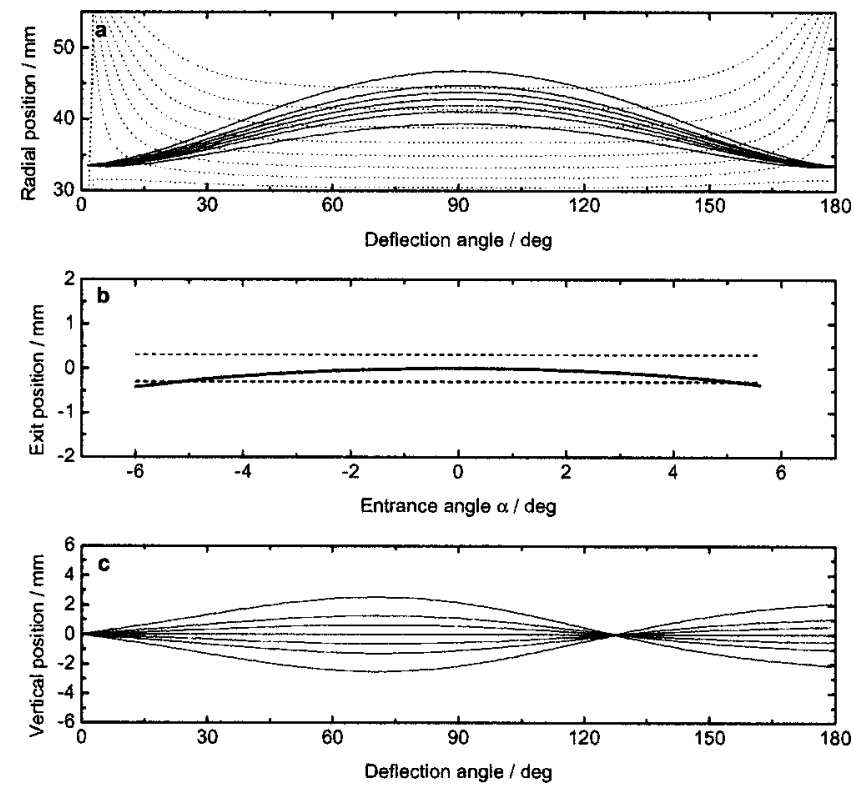

FIG. 5. Trajectories in the $180^{\circ}$ deflector in the zero current limit (details as in Fig. 4). within the dispersion plane in the exit slit. This setting provides optimum conditions for transmission and resolution, as shown later. The trajectories for electrons emerging from the center of the entrance slit with angles $\alpha=0^{\circ}, \pm 1^{\circ}, \pm 2^{\circ}$, and $\pm 4^{\circ}$ to the central trajectory are displayed in Figs. 4(a) and 5(a). In Figs. 4(b) and 5(b) the displacement from the central path in the exit slit $y(\alpha)$ is shown as a function of the angle $\alpha$. The first derivative $\partial y / \partial \alpha$ vanishes at $\alpha=0^{\circ}$ which indicates a first order focus at $90^{\circ}$ and $180^{\circ}$, respectively. $y(\alpha)$ can be fit to an expansion $y=-y_{0}-c_{\alpha \alpha} \alpha^{2}-c_{\alpha \alpha \alpha \alpha} \alpha^{4} \cdots$ in which the $c_{\alpha \alpha}$ and $c_{\alpha \alpha \alpha \alpha}$ are aberration coefficients and $y_{0}$ is the displacement of the trajectory from the center path in the entrance slit. The minus sign $y_{0}$ reflects the magnification -1 . The aberration coefficients are significantly smaller than for the cylindrical and spherical deflectors which is owed to the concave curvature of the deflector plates perpendicular to the dispersion plane. ${ }^{14}$

The trajectories in the sagittal plane are displayed for the entrance angles $\beta=0^{\circ}, \pm 1^{\circ}, \pm 2^{\circ}$, and $\pm 4^{\circ}$ in Figs. 4(c) and $5(\mathrm{c})$. Within the $90^{\circ}$ deflector one has no focusing at all. The trajectories diverge with an even larger angle $\beta$ at the exit slit than they had initially. Within the $180^{\circ}$ deflector one has an overfocusing: the focus is far ahead of the exit slit, approximately at $125^{\circ}$. Our initial hope was that beam divergence in the $90^{\circ}$ deflector could be compensated by the overfocusing of the $180^{\circ}$ deflector such that in total a stigmatic focus would be achieved at the exit slit. In order to investigate this proposition we fed the $180^{\circ}$ deflector with the trajectories exiting from the $90^{\circ}$ deflector. However, still an overfocusing was obtained and thus no stigmatic image at the exit slit. The overfocusing is, however, welcome as overfocusing deflectors better accommodate larger space charges due to high currents loads: The space charge always causes the beam to diverge, both in the dispersion plane and in the sagittal plane, whereby the foci in the two planes are shifted to larger deflection angles. In order to keep the focus in the dispersion plane at the exit slit the bias $U_{B}$ applied to top and bottom cover plates has to be changed to a more positive value. This in turn shifts the focus in the sagittal plane even further to larger deflection angles, and hence the overfocusing is reduced by the space charge. By playing with the design parameters one can design a spectrometer which focuses stigmatically under space charge load, albeit not without considerable aberrations.

In order to study the full transmission properties of the spectrometer under space charge conditions we proceeded as follows. In a first step the properties of the $90^{\circ}$ deflector are calculated self-consistently in the presence of the space charge as caused by the electrons supplied by the cathode (see above). An energy spread of $200 \mathrm{meV}$ of the electrons emitted from the cathode is assumed. The feeding current is set to $1 \mu \mathrm{A}$. The angular aperture is $\alpha=\beta= \pm 4^{\circ}$. The deflecting voltage in the $90^{\circ}$ deflector is $6 \mathrm{~V}$ corresponding to a pass energy of approximately $2.7 \mathrm{eV}$. The bias on top and bottom cover plates are set to have a focus in the dispersion plane. Transmission and resolution of the $90^{\circ}$ deflector are studied by feeding $10^{4}$ electrons with a particular energy into the entrance slit. The energy is then varied and the fraction of electrons emerging the exit slit is recorded as a function of 


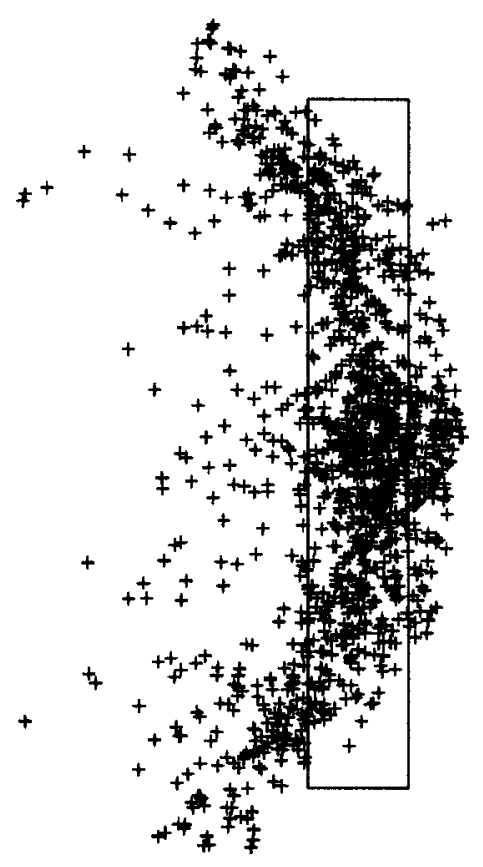

FIG. 6. Position (denoted as crosses) at which the electron hits the exit slit (rectangle) of the $180^{\circ}$ deflector for both deflectors operating under space charge conditions and for potential settings which yield the best performance. The crosses represent the image of the entrance slit. The 0.6 $\times 6 \mathrm{~mm}^{2}$ exit slit is marked as a rectangle.

energy. The full width at half maximum $\Delta E_{90^{\circ}}$ of that transmission curve and the beam parameters of the emerging electrons in the maximum of the transmission function are then used to feed the $180^{\circ}$ deflector in which the deflection voltage was $2 \mathrm{~V}$ (corresponding to a pass energy of about 0.9 $\mathrm{eV})$. Again the space charge potential is calculated with the energy spread of the beam entering the $180^{\circ}$ deflector. Thereafter, the transmission is calculated as a function of energy using the spatial and angular distribution of the electrons emerging from the $90^{\circ}$ deflector. The position of the trajectories on the exit slit of the $180^{\circ}$ deflector for electrons with an energy corresponding to the maximum transmission of the device are displayed in Fig. 6 as crosses together with the rectangular exit slit. While the image is not perfect (a perfect deflector would have all crosses inside the rectangle) the quality of the image is such that about $50 \%$ of the electrons fall into the exit slit. A slightly better result would be obtained if the exit slit was made to match the curved shape of the image. In view of the problems one encounters in processing the curved beam further in the spectrometer we have abstained from using curved slits. The transmission as a function of energy is shown in Fig. 7. The curved image gives rise to a tail on the right-hand side of the energy distribution. Despite the relatively poor image the width of the energy distribution, $\Delta E_{\mathrm{FWHM}}=6.2 \mathrm{meV}$, is only about $20 \%$ larger than for the $146^{\circ}$ deflector. The reason is that the $180^{\circ}$ deflector has a larger energy dispersion which compensates the low imaging qualities to a good extent.

The results in Figs. 6 and 7 are obtained after optimizing the focus with respect to beams emerging from the center point in the entrance slit. A question which needs still further exploration is as to whether this routine also provides the optimum transmission and energy resolution for a bundle of

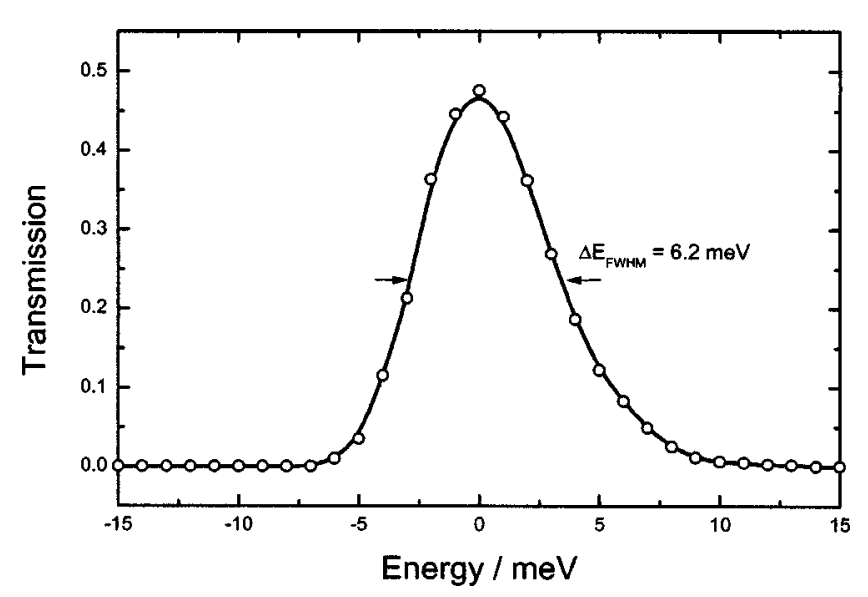

FIG. 7. Transmission of the $180^{\circ}$ deflector fed by the $90^{\circ}$ deflector as a function of the electron energy. The energy spread $\Delta E_{\mathrm{FWHM}}$ of $6.2 \mathrm{meV}$ is only $20 \%$ higher than for the standard $146^{\circ}$ deflector despite the relatively poor quality of the image (Fig. 6).

electrons which fills the entrance slit. This question is investigated by studying the energy width $\Delta E_{\mathrm{FWHM}}$, the transmission maximum, the total monochromatic current, and the "peak current" as a function of the bias voltage on the top and bottom cover plates. The peak current is defined as the current per energy window emerging from the deflector at the point of the maximum transmission (e.g., at 0.5 in Fig. 7). The quantities are plotted in Fig. 8 as a function of the bias voltage on the $180^{\circ}$ deflector. The energy spread has a clear, though shallow minimum which is practically at the bias voltage where the nominal focus was found with the trajectories emerging from the center of the entrance slit. Both transmission and the peak current have their maximum at the same point. This shows that the routine used to determine the optimum focus works satisfactory. Figure 8 demonstrates furthermore that the total current emerging from the deflector (which would be measured at the sample) displays no indication of an optimum focus at all. This is the reason that spectrometers can neither be optimized with respect to

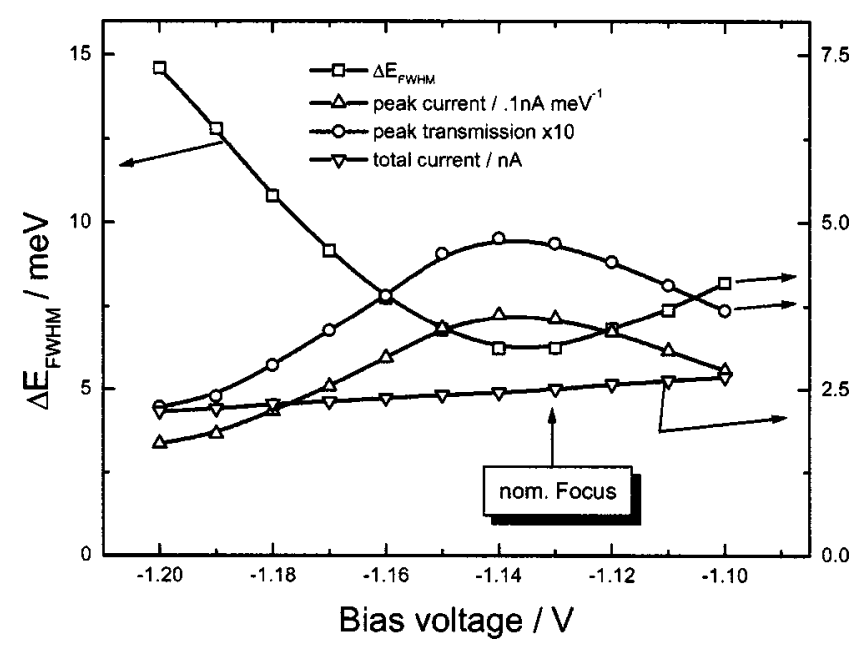

FIG. 8. Energy width $\Delta E_{\mathrm{FWHM}}$, the peak current, the peak transmission, and the total output current as a function of the bias voltage on the top and bottom cover plates of the $180^{\circ}$ deflector. The nominal focus is defined as the optimum focus of a bundle emerging at the center of the entrance slit. 


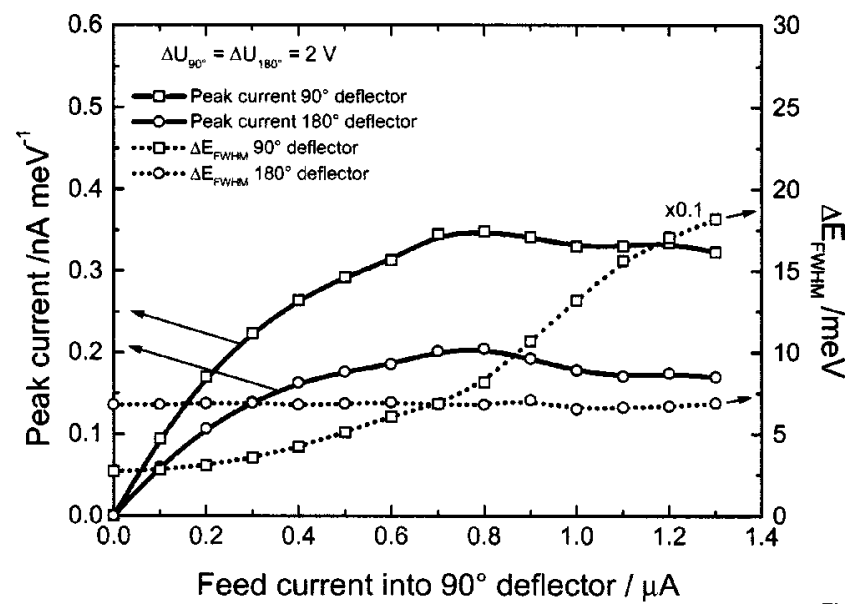

FIG. 9. Peak current and energy spread $\Delta E_{\mathrm{FWHM}}$ for the $90^{\circ}$ deflector and the $180^{\circ}$ deflector (fed by the $90^{\circ}$ deflector) as a function of the input current into the $90^{\circ}$ deflector. Deflection voltages are $\Delta U_{90^{\circ}}=\Delta U_{180^{\circ}}=2 \mathrm{~V}$.

applied potentials nor evaluated in their qualities by observing the current at the sample.

\section{PERFORMANCE OF THE SPECTROMETER}

As demonstrated with Fig. 8, the peak current is a good measure of the performance of a spectrometer. The particular virtue in referring to the peak current in comparing performances as well as in optimizing spectrometer designs is that the peak current anywhere along the electron path through the spectrometer can only be less than it was in the preceding spectrometer section. Hence the peak current is a single quantity whereby the performance, e.g., of the first monochromator can be checked already without investigating the processing of the beam in spectrometer sections further down the path. We have therefore studied the peak current and the energy spread $\Delta E_{\mathrm{FWHM}}$ for the $90^{\circ}$ deflector and the $180^{\circ}$ deflector, as fed by the $90^{\circ}$ deflector as a function of the input current of the $90^{\circ}$ deflector. We have considered two cases, one where the $90^{\circ}$ deflector operates with the same deflection voltage (here $2 \mathrm{~V}$ ), and a second where a deflection voltage of $6 \mathrm{~V}$ is applied to the $90^{\circ}$ deflector so that the $90^{\circ}$ deflector operated as a retarding deflector. ${ }^{1}$ The energy width of the feed beam to the $90^{\circ}$ deflector (used to calculate the space charge) is $200 \mathrm{meV}$, as before. The space charge is taken into account self-consistently as described in Sec. II. The data calculated for the combination of the $90^{\circ} / 180^{\circ}$ deflectors are obtained by feeding the $180^{\circ}$ deflector with exactly the trajectories as they emerged from the $90^{\circ}$ deflector, as described above. The results are displayed in Figs. 9 and 10 for $\Delta U_{90^{\circ}}=\Delta U_{180^{\circ}}=2 \mathrm{~V}$ and $\Delta U_{90^{\circ}}=6 \mathrm{~V}$, respectively. Both the peak currents emerging from the $90^{\circ}$ deflector and from the combination of the two deflectors are initially proportional to the input current and level-off for higher input currents. In the case where $\Delta U_{90^{\circ}}=\Delta U_{180^{\circ}}$ the peak current appears to pass through a shallow maximum. The saturation of the peak current is caused by the decreasing performance of the $90^{\circ}$ deflector due to the heavy current load. The decreasing performance is manifest also in the increase of the energy spread of the $90^{\circ}$ deflector (Figs. 9 and 10). The energy spread of the $180^{\circ}$ deflector stays nearly unaffected

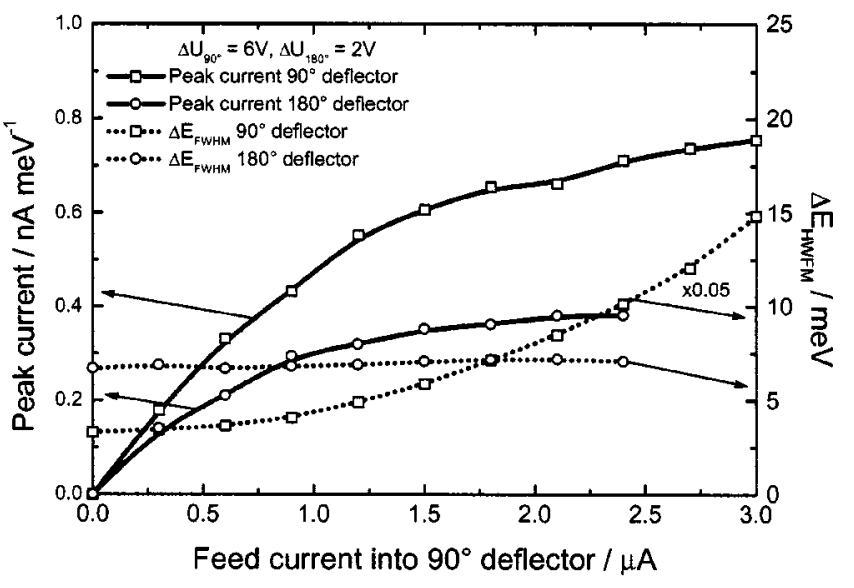

FIG. 10. Peak current and energy spread $\Delta E_{\mathrm{FWHM}}$ for the $90^{\circ}$ deflector and the $180^{\circ}$ deflector (fed by the $90^{\circ}$ deflector) as a function of the input current into the $90^{\circ}$ deflector. Deflection voltages are $\Delta U_{90^{\circ}}=6 \mathrm{~V}$ and $\Delta U_{180^{\circ}}$ $=2 \mathrm{~V}$. Hence the $90^{\circ}$ deflector operates as a retarding deflector. The resolution of the $90^{\circ}$ deflector at $3 \mu \mathrm{A}\left(\Delta E_{\mathrm{FWHM}}=300 \mathrm{meV}\right)$ is calculated without considering the finite energy spread of the cathode ( $\Delta E_{\mathrm{FWHM}}$ $=200 \mathrm{meV}$ ).

because of the lower space charge aberrations in the $180^{\circ}$ deflector. The peak current obtained in the retarding case is significantly higher than for the case where both deflectors operate at the same path energy. Hence it is advantageous to work with the retardation, provided that the cathode system is able to deliver the higher input current required for the retarding combination. The energy resolution is practically not affected by operating the $90^{\circ}$ deflector in the retarding mode.

As a final step in the analysis of the new spectrometer we calculate the monochromatic current as measured in the detector with the beam shooting directly into the analyzer and compare it to experimental measurements. To a good approximation the monochromatic $I_{M}$ current scales with the resolution of the monochromator $\Delta E_{M}$ and analyzer $\Delta E_{A}$ as

$$
I_{M} \propto \Delta E_{M}^{3 / 2} \Delta E_{A} .
$$

The exponent $3 / 2$ arises from the space charge limited current and the scaling with $\Delta E_{A}$ is because a smaller $\Delta E_{A}$ cuts out a smaller section of the energy distribution of the beam delivered by the monochromator. Assuming Gaussian transmission functions the total resolution of the spectrometer is

$$
\Delta E_{\mathrm{tot}}=\left(\Delta E_{M}^{2}+\Delta E_{A}^{2}\right)^{1 / 2} .
$$

The maximum current $I_{M}$ at a given $\Delta E_{\text {tot }}$ is easily calculated from Eqs. (1) and (2) to occur if

$$
\Delta E_{A}=\sqrt{\frac{2}{3}} \Delta E_{M}
$$

or

$$
\Delta E_{\mathrm{tot}}=\sqrt{\frac{5}{3}} \Delta E_{M} .
$$

Assuming again Gaussian transmission functions the fraction of the input current transmitted by the analyzer is 


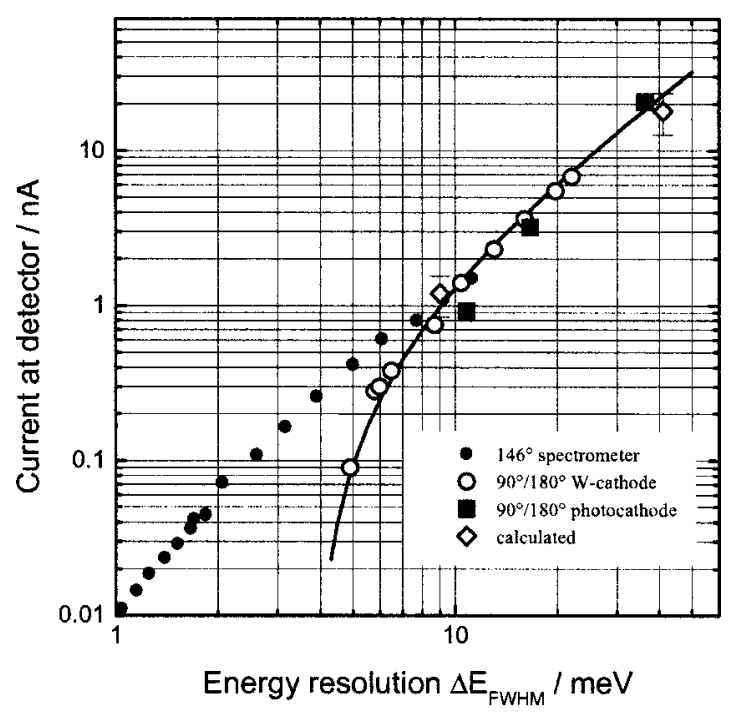

FIG. 11. Monochromatic current at the detector as measured in the "straight through" position for the best available spectrometer (Ref. 2) (closed circles) which was designed for highest resolution and the spin spectrometer (open circles and closed squares) characterized by a trade-off of resolution in favor of higher currents to match the requirements of measuring spin flip transitions. The open diamonds are calculated from the simulations.

$$
T_{A}=T_{\max } \sqrt{\frac{\Delta E_{M} \Delta E_{A}}{\Delta E_{M}^{2}+\Delta E_{A}^{2}}},
$$

in which $T_{\max }$ is the transmission at the maximum of the transmission function of the analyzer (compare to Fig. 7). After inserting $T_{\max } \approx 0.7$ for the $146^{\circ}$ analyzer and Eq. (3) one obtains $T_{A} \approx 0.5$. The transmission for the lens system between monochromator exit and the analyzer entrance slit is about $80 \%,{ }^{1}$ which makes the current at the detector amount to about $40 \%$ of the current at the sample. The overall resolution is reduced according to Eq. (4). With these considerations the current at the detector can be calculated as a function of the overall resolution once the current emerging from the monochromator versus resolution is known. We have performed the corresponding calculations for two potential settings of the spectrometer: $\Delta U_{90^{\circ}}=6 \mathrm{~V}, \Delta U_{180^{\circ}}=2 \mathrm{~V}$, and $\Delta U_{90^{\circ}}=\Delta U_{180^{\circ}}=9 \mathrm{~V}$. The results for the current at the detector are shown in Fig. 11 as open diamonds. The open circles and the closed squares are measurements with a tungsten cathode and the photocathode, respectively. The closed circles represent data of the best known spectrometer featur- ing the $146^{\circ}$ aberration compensated deflectors. Figure 11 demonstrates that the numerical calculations match the experimental results quite well. Furthermore, the spectrometer design for spin dependent scattering does not fall short in performance compared to the best spectrometer known so far. The lower resolution limit for the spin spectrometer of about $4 \mathrm{meV}$ rather than $0.3 \mathrm{meV}$ for the $146^{\circ}$ spectrometer is a consequence of the fact that the spin spectrometer features larger slits and was designed to operate with area-rather than tip-cathodes.

Finally, we compare the present design in its performance with the proposal of Vattuone et al. ${ }^{15}$ to use a $90^{\circ}$ deflector only. For this deflector Vattuone et al. calculate a monochromatic current at the sample of $0.2 \mathrm{nA}$ for a monochromator resolution of $10 \mathrm{meV}$. Scaled to the current at the detector and the resolution of the complete spectrometer this corresponds to $I_{M}=0.08 \mathrm{nA}$ at $\Delta E_{\text {tot }}=13 \mathrm{meV}$ which is a factor of 30 lower than in the design described here. The reasons for the inferior performance of a spectrometer having only a $90^{\circ}$ monochromator are the poor energy dispersion and focusing properties of the $90^{\circ}$ deflector.

\section{ACKNOWLEDGMENT}

Partial financial support by the Fond der Chemischen Industrie for H. I. is highly appreciated.

${ }^{1} \mathrm{H}$. Ibach, Electron Energy Loss Spectroscopy-The Technology of High Performance (Springer, Heidelberg, 1991).

${ }^{2}$ H. Ibach et al., Surf. Sci. 269/270, 94 (1992).

${ }^{3}$ H. Ibach, M. Balden, and S. Lehwald, J. Chem. Soc., Faraday Trans. 92, 4771 (1996).

${ }^{4}$ C. E. Kuyatt and J. A. Simpson, Rev. Sci. Instrum. 38, 103 (1967).

${ }^{5}$ P. Grünberg et al., J. Magn. Magn. Mater. 28, 319 (1982).

${ }^{6}$ R. E. Camley, P. Grünberg, and C. M. Mayr, Phys. Rev. B 26, 2609 (1982).

${ }^{7}$ M. P. Gokhale, A. Ormeci, and D. L. Mills, Phys. Rev. B 46, 8978 (1992).

${ }^{8}$ M. Plihal and D. L. Mills, Phys. Rev. B 58, 14407 (1998).

${ }^{9}$ M. Plihal, D. L. Mills, and J. Kirschner, Phys. Rev. Lett. 82, 2579 (1999).

${ }^{10}$ D. T. Pierce et al., Rev. Sci. Instrum. 51, 478 (1980).

${ }^{11}$ J. Kirschner, H. P. Oepen, and H. Ibach, Appl. Phys. A: Solids Surf. A30, 177 (1983).

${ }^{12}$ H. Ibach and D. L. Mills, Electron Energy Loss Spectroscopy and Surface Vibrations (Academic, New York, 1982).

${ }^{13}$ J. Kirschner, D. Rebensdorff, and H. Ibach, Phys. Rev. Lett. 53, 698 (1984).

${ }^{14}$ H. Ibach, J. Electron Spectrosc. Relat. Phenom. 64/65, 819 (1993).

${ }^{15}$ L. Vattuone and M. Rocca, Rev. Sci. Instrum. 73, 3861 (2002).

${ }^{16} \mathrm{H}$. Ibach, D. Bruchmann, and S. Lehwald, in European Patent EP 0554 814 B1, Germany, 2001 